BULLETIN OF THE

AMERICAN MATHEMATICAL SOCIETY

Volume 77, Number 1, January 1971

\title{
TRANSLATION-INVARIANT LINEAR FORMS \\ AND A FORMULA FOR THE DIRAC MEASURE
}

\author{
BY GARY H. MEISTERS ${ }^{1}$
}

Communicated by Peter D. Lax, July 27, 1970

Following Schwartz [2] we denote by $\mathcal{D}, \varepsilon$ and $\delta$ the complex vector spaces of all complex-valued infinitely differentiable functions $\phi$ on $R^{n}$ where the functions of $D$ have compact supports, the functions of $\varepsilon$ have arbitrary supports, and the functions of $\delta$ (along with all their derivatives) are rapidly decreasing at infinity. We equip each of these spaces with its usual locally convex topology. These spaces and their duals $D^{\prime}, \mathcal{E}^{\prime}$ and $\mathcal{S}^{\prime}$ are translation-invariant in the sense that the translated function (or distribution) $\phi_{h}(t) \equiv \phi(t-h)$ belongs to the space whenever $\phi$ does. We say that a (not necessarily continuous) linear form $L$ on any of these spaces is "translation-invariant" if $L\left(\phi_{h}\right)=L(\phi)$ for all $\phi$ in the domain space and for all $h$ in $R^{n}$. It is, of course, well known what the conlinuous translation-invariant linear forms on these spaces are like; namely, they are either identically zero or a constant multiple of integration over $R^{n}$.

The purpose of this paper is to announce that there exists no discontinuous translation-invariant linear form on any of the six spaces $D, \varepsilon, S, D^{\prime}, \mathcal{E}^{\prime}$ or $\delta^{\prime}$. That is, integration over $R^{n}$ in the spaces $D$ and $\mathcal{E}^{\prime}$ can be characterized (up to a multiplicative constant) simply as a translation-invariant linear form. Furthermore, we obtain this result as a simple consequence of a resolution of the first derivative of the Dirac measure $\delta$ (on the real line $R$ ) into a sum of two finite differences of distributions of compact support. We state this as our main result.

THEOREM 1. If $\alpha$ and $\beta$ are nonzero real numbers such that $\alpha / \beta$ is irrational and not a Liouville transcendental, then there exist two (necessarily distinct) distributions $S$ and $T$ on $R$, both with compact

AMS 1970 subject classifications. Primary 46F10, 39A05, 46H10; Secondary $10 \mathrm{~F} 25,42 \mathrm{~A} 68,28 \mathrm{~A} 30$.

Key words and phrases. Infinitely differentiable functions, translation-invariant linear forms, Dirac measure, distributions with compact supports, finite differences, algebraic irrationals, convolution, tensor product, entire functions, Paley-WienerSchwartz Theorem, Fourier transforms, Liouville transcendental.

1 This research was supported in part by NSF Grant GP-11605. 
supports, such that

$$
\delta^{\prime}=S-S_{\alpha}+T-T_{\beta}
$$

and conversely.

Here $S_{\alpha}$ denotes the translate of the distribution $S$ by the real number $\alpha$, and is defined by the equation $\left\langle S_{\alpha}, \phi\right\rangle=\left\langle S, \phi_{-\alpha}\right\rangle$ for all test functions $\phi$. Furthermore, $S$ and $T$ can be chosen to have order 2 (at least when $\alpha / \beta$ is a quadratic irrational) but can not have any lower order.

Note that if $\phi$ belongs to any of the spaces $D, \mathcal{E}, \mathcal{S}$, or their duals, then the convolution products $u \equiv \phi * S$ and $v \equiv \phi * T$ exist and belong to the same space as $\phi$. Thus by convolution with $\phi$ formula (1) yields

$$
\phi^{\prime}=u-u_{\alpha}+v-v_{\beta},
$$

with $\phi, u$ and $v$ all in the same space. Equations (1) and (2) can be generalized to $R^{n}$ (for $n \geqq 2$ ) by means of the tensor product of distributions. Equation (2), or its generalization to $R^{n}$, implies that the null space $\Re$ of a translation-invariant linear form $L$ (on $\mathscr{D}\left(R^{n}\right)$, for example) must contain the null space of integration. Consequently, there must exist a complex constant $c$ such that $L(\phi)$ $=c \cdot \int_{R_{n}} \phi(t) d t$ for all $\phi$ in $D\left(R^{n}\right)$.

The details of the proofs of Theorem 1 and related results, and the proofs of the other statements made above concerning $S$ and $T$ in formula (1), are to appear in J. Functional Analysis. We only indicate here the main steps in the proof of Theorem 1. According to Liouville (see [1, Theorem 191, p. 161]), if $\alpha / \beta$ is an algebraic real number of degree ' $\geqq 2$, there exists a positive constant $K$ such that, for all nonzero integers $k$,

$$
|1-\exp [-2 \pi i \alpha k / \beta]|^{-1} \leqq K|k|^{\ell-1} .
$$

We shall consider here only the case that $\ell=2(\alpha / \beta$ is a quadratic irrational). We define an entire analytic function $\hat{S}(z)$ by the expression

$$
\left(-z / 4 \pi^{2}\right)(1-\exp [-2 \pi i \beta z])^{3} \sum_{-\infty}^{+\infty}(1-\exp [-2 \pi i \alpha k / \beta])^{-1}(\beta z-k)^{-3} \text {. }
$$

Then $\hat{S}$ can be shown to satisfy

$$
\hat{S}(k / \beta)=(2 \pi i k / \beta)(1-\exp [-2 \pi i \alpha k / \beta])^{-1},
$$

for all nonzero integers $k$. Also the inequality (3) allows us to establish the following estimate for $\hat{S}(z)$.

$$
|S(z)| \leqq C|z|(1+|z|) e^{b|y|}, \text { for all } z=x+i y,
$$


for some positive constants $C$ and $b$. It follows that

$$
\hat{T}(z) \equiv[2 \pi i z-\hat{S}(z)(1-\exp [-2 \pi i \alpha z])](1-\exp [-2 \pi i \beta z])^{-1}
$$

is also entire and can be shown to satisfy the estimate

$$
|\hat{T}(z)| \leqq B|z|(1+|z|) e^{c|y|}, \text { for all } z=x+i y,
$$

for some constants $B$ and $c$. Now the inequalities (4) and (6) imply according to the Paley-Wiener-Schwartz Theorem (see [2, Théorème XVI, p. 272]) that $\hat{S}$ and $\hat{T}$ are the Fourier transforms of two distributions $S$ and $T$ of compact support on the real line $R$. But then taking inverse Fourier transforms of both sides of equation (5), after first multiplying through by the factor $\left(1-e^{-2 \pi i \beta z}\right)$, we obtain formula (1) of Theorem 1.

\section{REFERENCES}

1. G. H. Hardy and E. M. Wright, An introduction to the theory of numbers, 3rd ed., Clarendon Press, Oxford, 1954. MR 16, 673.

2. Laurent Schwartz, Theorie des distributions, 2nd ed., Publ. Inst. Math. Univ. Strasbourg, nos. 9, 10, Hermann, Paris, 1966. MR 35 \#730.

University of Colorado, Boulder, Colorado 80302 